\title{
Developing CRS iterative methods for periodic Sylvester matrix equation
}

\author{
Linjie Chen ${ }^{1}$ and Changfeng $\mathrm{Ma}^{{ }^{*}}$
}

\section{"Correspondence:}

macf@fjnu.edu.cn

${ }^{1}$ College of Mathematics and

Informatics, Fujian Normal

University, Fuzhou, P.R. China

\section{Introduction}

We consider the iterative solution of the periodic Sylvester matrix equation

$$
A_{j} X_{j} B_{j}+C_{j} X_{j+1} D_{j}=E_{j} \quad \text { for } j=1,2, \ldots,
$$

where the coefficient matrices $A_{j}, B_{j}, C_{j}, D_{j}, E_{j} \in \mathbf{R}^{m \times m}$ and the solutions $X_{j} \in \mathbf{R}^{m \times m}$ are periodic with period $\lambda$, that is, $A_{j+\lambda}=A_{j}, B_{j+\lambda}=B_{j}, C_{j+\lambda}=C_{j}, D_{j+\lambda}=D_{j}, E_{j+\lambda}=E_{j}$, and $X_{j+\lambda}=$ $X_{j}$. The periodic Sylvester matrix equation (1.1) attracts considerable attention because it comes from a variety of fields of control theory and applied mathematics [1-12].

In recent years, many efficient iterative methods have been proposed to solve the periodic Sylvester matrix equation (1.1). For example, Hajarian [13, 14] developed the conjugate gradient squared (CGS), biconjugate gradient stabilized (BiCGSTAB) and biconjugate residual methods for solving the periodic Sylvester matrix equation (1.1). Lv and Zhang [15] proposed a new kind of iterative algorithm for constructing the least square solution for the periodic Sylvester matrix equation. Hajarian [16] studied the biconjugate $A$-orthogonal residual and conjugate $A$-orthogonal residual squared (CORS) methods for solving coupled periodic Sylvester matrix equation, and so forth; see [17-28] and the references therein.

As we know, by applying Kronecker product and vectorization operator, some iterative algorithms for solving linear system $A x=b$ can be extended to solve linear matrix equa-

(c) The Author(s) 2019. This article is distributed under the terms of the Creative Commons Attribution 4.0 International License (http://creativecommons.org/licenses/by/4.0/), which permits unrestricted use, distribution, and reproduction in any medium, provided you give appropriate credit to the original author(s) and the source, provide a link to the Creative Commons license, and indicate if changes were made. 
tions. Recently, Sogabe et al. [29] proposed a conjugate residual squared (CRS) method for solving linear systems $A x=b$ with nonsymmetric coefficient matrix. Independently, Zhang et al. [30] presented another form of CRS method. It can be proved that these CRS methods are mathematically equivalent. Chen and Ma [31] used the matrix CRS iterative method to solve a class of coupled Sylvester-transpose matrix equations. In this work, we obtain a matrix form of the CRS methods for solving the periodic Sylvester matrix equation (1.1).

The rest of this paper is organized as follows. In Sect. 2, we extend the CRS methods to solve the periodic Sylvester matrix equation (1.1). We give some numerical examples and comparison results in Sect. 3. In Sect. 4, we draw a brief conclusion.

Throughout this paper, we use the following notations. The set of all real $m$-vectors and the set of all $m \times n$ real matrices are denoted by $\mathbf{R}^{m}$ and $\mathbf{R}^{m \times n}$, respectively. The usual inner product in $\mathbf{R}^{m}$ is denoted by $(u, v)$ for $u, v \in \mathbf{R}^{m}$. For a matrix $A \in \mathbf{R}^{m \times n}$, we denote its trace and transpose by $\operatorname{tr}(A)$ and $A^{T}$, respectively. The inner product of $A \in \mathbf{R}^{m \times n}$ and $B \in \mathbf{R}^{m \times n}$ is defined by $\langle A, B\rangle=\operatorname{tr}\left(B^{T} A\right)$. Then the norm of a matrix generated by this inner product is the matrix Frobenius norm $\|\cdot\|$. For a matrix $A \in \mathbf{R}^{m \times n}$, the vectorization operator is defined as $\operatorname{vec}(A)=\left(\begin{array}{llll}a_{1}^{T} & a_{2}^{T} & \cdots & a_{n}^{T}\end{array}\right)^{T}$, where $a_{i}$ is the $i$ th column of $A$. The Kronecker product of matrices $A=\left[a_{i j}\right] \in \mathbf{R}^{m \times n}$ and $B \in \mathbf{R}^{p \times q}$ is defined as $A \otimes B=\left[a_{i j} B\right] \in \mathbf{R}^{m p \times n q}$. For matrices $A, B$, and $X$ of appropriate dimensions, we have the following well-known property related to the Kronecker product and vectorization operator:

$$
\operatorname{vec}(A X B)=\left(B^{T} \otimes A\right) \operatorname{vec}(X) .
$$

\section{Matrix forms of the CRS iterative methods}

In this section, we first briefly recall the CRS iterative methods for solving a large sparse nonsymmetric linear system $A x=b$, where $A \in \mathbf{R}^{N \times N}$ and $x, b \in \mathbf{R}^{N}$. As described in the introduction, the CRS iterative methods are presented in [29] and [30], which are summarized as the following Algorithms 2.1 and 2.2, respectively. For more detail about the CRS methods, see [32-34].

Algorithm 2.1 (The first form of CRS method (CRS1) [29])

1. $x_{0}$ is an initial guess; $r_{0}=b-A x_{0}$; choose $r_{0}^{*}$ (for example, $r_{0}^{*}=r_{0}$ ).

2. Set $e_{0}=r_{0}, d_{0}=A e_{0}, \beta_{-1}=0$. Let $t=A^{T} r_{0}^{*}$.

3. For $n=0,1, \ldots$, until convergence Do:

$$
\begin{aligned}
& s_{n}=d_{n}+\beta_{n-1}\left(f_{n-1}+\beta_{n-1} s_{n-1}\right) ; \\
& \alpha_{n}=\left(t, r_{n}\right) /\left(t, s_{n}\right) ; \\
& h_{n}=e_{n}-\alpha_{n} s_{n} ; \\
& f_{n}=d_{n}-\alpha_{n} A s_{n} ; \\
& x_{n+1}=x_{n}+\alpha_{n}\left(e_{n}+h_{n}\right) ; \\
& r_{n+1}=r_{n}-\alpha_{n}\left(d_{n}+f_{n}\right) ; \\
& \beta_{n}=\left(t, r_{n+1}\right) /\left(t, r_{n}\right) ; \\
& e_{n+1}=r_{n+1}+\beta_{n} h_{n}
\end{aligned}
$$




$$
d_{n+1}=A r_{n+1}+\beta_{n} f_{n}
$$

\section{EndDo.}

Algorithm 2.2 (The second form of CRS method (CRS2) [30])

1. Compute $r_{0}=b-A x_{0}$; choose $r_{0}^{*}$ such that $\left(A r_{0}, r_{0}^{*}\right) \neq 0$ (for example, $r_{0}^{*}=r_{0}$ ).

2. Set $p_{0}=u_{0}=r_{0}$. Let $t=A^{T} r_{0}^{*}$.

3. For $n=0,1, \ldots$, until convergence Do:

$$
\begin{aligned}
& \alpha_{n}=\left(t, r_{n}\right) /\left(t, A p_{n}\right) ; \\
& q_{n}=u_{n}-\alpha_{n} A p_{n} ; \\
& x_{n+1}=x_{n}+\alpha_{n}\left(u_{n}+q_{n}\right) ; \\
& r_{n+1}=r_{n}-\alpha_{n} A\left(u_{n}+q_{n}\right) ; \\
& \beta_{n}=\left(t, r_{n+1}\right) /\left(t, r_{n}\right) ; \\
& u_{n+1}=r_{n+1}+\beta_{n} q_{n} ; \\
& p_{n+1}=u_{n+1}+\beta_{n}\left(q_{n}+\beta_{n} p_{n}\right) ;
\end{aligned}
$$

\section{EndDo.}

Let

$$
h_{n} \leftrightarrow q_{n}, \quad e_{n} \leftrightarrow u_{n}, \quad s_{n} \leftrightarrow A p_{n}, \quad d_{n} \leftrightarrow A u_{n}, \quad f_{n} \leftrightarrow A q_{n} .
$$

Then we can verify that CRS1 and CRS2 are mathematically equivalent. The CRS methods were proposed mainly to avoid using the transpose of $A$ in the BiCR algorithm and gain a faster convergence for roughly the same computational costs [30]. Indeed, in many cases, the CRS methods converge twice as fast as the BiCR method [33, 35]. On the other hand, the BiCR method can be derived from the preconditioned conjugate residual (CR) method [36]. Furthermore, the CR and conjugate gradient (CG) methods exhibit typically similar convergence [37]. In exact arithmetic, they terminate after a finite number of iterations. In conclusion, we can expect that the CRS methods also terminate after a finite number of iterations in exact arithmetic.

In the following, we want to use the CRS algorithms to solve the periodic Sylvester matrix equations (1.1). For this purpose, we can easily show that the periodic Sylvester matrix equation (1.1) is equivalent to the following generalized Sylvester matrix equation [13]:

$$
\mathrm{AXB}+\mathrm{CXD}=\mathbf{E},
$$

where

$$
\mathbf{A}=\left[\begin{array}{cccc}
0 & \cdots & 0 & A_{1} \\
A_{2} & & & 0 \\
& \ddots & & \vdots \\
0 & & A_{\lambda} & 0
\end{array}\right], \quad \mathbf{B}=\left[\begin{array}{cccc}
0 & B_{2} & & 0 \\
\vdots & & \ddots & \\
0 & & & B_{\lambda} \\
B_{1} & 0 & \cdots & 0
\end{array}\right]
$$




$$
\begin{aligned}
& \mathbf{C}=\operatorname{diag}\left[C_{1}, C_{2}, \ldots, C_{\lambda}\right], \quad \mathbf{D}=\operatorname{diag}\left[D_{1}, D_{2}, \ldots, D_{\lambda}\right], \\
& \mathbf{E}=\operatorname{diag}\left[E_{1}, E_{2}, \ldots, E_{\lambda}\right], \quad \mathbf{X}=\operatorname{diag}\left[X_{2}, X_{3}, \ldots, X_{\lambda}, X_{1}\right] .
\end{aligned}
$$

Then we need to transform the generalized Sylvester matrix equations (2.1) into a linear system $A x=b$. We should mention that the following derivation borrows much of that used in [13].

By applying the Kronecker product and vectorization operator we can change the generalized Sylvester matrix equations (2.1) into the following linear system of equations:

$$
\left(\mathbf{B}^{T} \otimes \mathbf{A}+\mathbf{D}^{T} \otimes \mathbf{C}\right) \operatorname{vec}(\mathbf{X})=\operatorname{vec}(\mathbf{E})
$$

Denote

$$
A:=\mathbf{B}^{T} \otimes \mathbf{A}+\mathbf{D}^{T} \otimes \mathbf{C}, \quad x:=\operatorname{vec}(\mathbf{X}), \quad b:=\operatorname{vec}(\mathbf{E}) .
$$

Then (2.2) can be written as

$$
A x=b,
$$

where $A \in R^{\lambda^{2} m^{2} \times \lambda^{2} m^{2}}$ and $x, b \in R^{\lambda^{2} m^{2}}$. Then we are in position to present the matrix forms of Algorithms 2.1 and 2.2 for solving the generalized Sylvester matrix equation (2.1), and we just discuss Algorithm 2.1 in detail since the discussion of Algorithm 2.2 is similar.

From Algorithm 2.1 and the linear system of equation (2.2) we have

$$
\begin{aligned}
& r_{0}=b-A x_{0} \rightarrow r_{0}=\operatorname{vec}(\mathbf{E})-\left(\mathbf{B}^{T} \otimes \mathbf{A}+\mathbf{D}^{T} \otimes \mathbf{C}\right) x_{0}, \\
& d_{0}=A e_{0} \rightarrow d_{0}=\left(\mathbf{B}^{T} \otimes \mathbf{A}+\mathbf{D}^{T} \otimes \mathbf{C}\right) e_{0}, \\
& t=A^{T} r_{0}^{*} \rightarrow t=\left(\mathbf{B} \otimes \mathbf{A}^{T}+\mathbf{D} \otimes \mathbf{C}^{T}\right) r_{0}^{*}, \\
& A s_{n} \rightarrow\left(\mathbf{B}^{T} \otimes \mathbf{A}+\mathbf{D}^{T} \otimes \mathbf{C}\right) s_{n}, \\
& A r_{n+1} \rightarrow\left(\mathbf{B}^{T} \otimes \mathbf{A}+\mathbf{D}^{T} \otimes \mathbf{C}\right) r_{n+1} .
\end{aligned}
$$

According to (2.3)-(2.7), we define

$$
\begin{array}{lll}
x_{n}=\operatorname{vec}(\mathbf{X}(n)), & r_{n}=\operatorname{vec}(\mathbf{R}(n)), & s_{n}=\operatorname{vec}(\mathbf{S}(n)), \\
h_{n}=\operatorname{vec}(\mathbf{H}(n)), & f_{n}=\operatorname{vec}(\mathbf{F}(n)), & e_{n}=\operatorname{vec}(\mathbf{E}(n)), \\
d_{n}=\operatorname{vec}(\mathbf{D}(n)), & r_{0}^{*}=\operatorname{vec}\left(\mathbf{R}^{*}(0)\right), & t=\operatorname{vec}(\mathbf{T}),
\end{array}
$$

where $\mathbf{X}(n), \mathbf{R}(n), \mathbf{S}(n), \mathbf{H}(n), \mathbf{F}(n), \mathbf{E}(n), \mathbf{D}(n), \mathbf{R}^{*}(0), \mathbf{T} \in \mathbf{R}^{\lambda m \times \lambda m}$ for $n=0,1,2, \ldots$ Substituting (2.8)-(2.10) into (2.3)-(2.7), we get

$$
\begin{aligned}
& \operatorname{vec}(\mathbf{R}(0))=\operatorname{vec}(\mathbf{E})-\left(\mathbf{B}^{T} \otimes \mathbf{A}+\mathbf{D}^{T} \otimes \mathbf{C}\right) \operatorname{vec}(\mathbf{X}(0)), \\
& \operatorname{vec}(\mathbf{D}(0))=\left(\mathbf{B}^{T} \otimes \mathbf{A}+\mathbf{D}^{T} \otimes \mathbf{C}\right) \operatorname{vec}(\mathbf{E}(0)), \\
& \operatorname{vec}(\mathbf{T})=\left(\mathbf{B} \otimes \mathbf{A}^{T}+\mathbf{D} \otimes \mathbf{C}^{T}\right) \operatorname{vec}\left(\mathbf{R}^{*}(0)\right),
\end{aligned}
$$




$$
A s_{n}=\left(\mathbf{B}^{T} \otimes \mathbf{A}+\mathbf{D}^{T} \otimes \mathbf{C}\right) \operatorname{vec}(\mathbf{S}(n))
$$

and

$$
A r_{n+1}=\left(\mathbf{B}^{T} \otimes \mathbf{A}+\mathbf{D}^{T} \otimes \mathbf{C}\right) \operatorname{vec}(\mathbf{R}(n+1))
$$

In addition, for the parameters $\alpha_{n}$ and $\beta_{n}$, we have

$$
\alpha_{n}=\langle\mathbf{R}(n), \mathbf{T}\rangle /\langle\mathbf{V}(n), \mathbf{T}\rangle
$$

and

$$
\beta_{n}=\langle\mathbf{R}(n+1), \mathbf{T}\rangle /\langle\mathbf{R}(n), \mathbf{T}\rangle
$$

From this discussion it follows that the matrix form of CRS1 method for solving the generalized Sylvester matrix equation (2.1) can be constructed as the following Algorithm 2.3. Analogously, the matrix form of CRS1 method for solving the generalized Sylvester matrix equation (2.1) is summarized as Algorithm 2.4.

Algorithm 2.3 (Matrix CRS1 method for solving (2.1))

1. Compute $\mathbf{R}(0)=\mathbf{E}-\mathbf{A X}(0) \mathbf{B}-\mathbf{C X}(0) \mathbf{D}$ for an initial guess $\mathbf{X}(0) \in \mathbf{R}^{\lambda m \times \lambda m}$. Set $\mathbf{R}^{*}(0)=\mathbf{E}(0)=\mathbf{R}(0)$ and $\mathbf{D}(0)=\mathbf{A E}(0) \mathbf{B}+\mathbf{C E}(0) \mathbf{D}$. Let $\beta_{-1}=0$.

2. Set $\mathbf{T}=\mathbf{A}^{T} \mathbf{R}^{*}(0) \mathbf{B}^{T}+\mathbf{C}^{T} \mathbf{R}^{*}(0) \mathbf{D}^{T}$.

3. For $n=0,1, \ldots$, until convergence Do:

$$
\begin{aligned}
& \mathbf{S}(n)=\mathbf{D}(n)+\beta_{n-1}\left(\mathbf{F}(n-1)+\beta_{n-1} \mathbf{S}(n-1)\right) ; \\
& \alpha_{n}=\langle\mathbf{R}(n), \mathbf{T}\rangle /\langle\mathbf{S}(n), \mathbf{T}\rangle ; \\
& \mathbf{H}(n)=\mathbf{E}(n)-\alpha_{n} \mathbf{S}(n) ; \\
& \mathbf{F}(n)=\mathbf{D}(n)-\alpha_{n}(\mathbf{A} \mathbf{S}(n) \mathbf{B}+\mathbf{C S}(n) \mathbf{D}) ; \\
& \mathbf{X}(n+1)=\mathbf{X}(n)+\alpha_{n}(\mathbf{E}(n)+\mathbf{H}(n)) ; \\
& \mathbf{R}(n+1)=\mathbf{R}(n)-\alpha_{n}(\mathbf{D}(n)+\mathbf{F}(n)) ; \\
& \beta_{n}=\langle\mathbf{R}(n+1), \mathbf{T}\rangle /\langle\mathbf{R}(n), \mathbf{T}\rangle ; \\
& \mathbf{E}(n+1)=\mathbf{R}(n+1)+\beta_{n} \mathbf{H}(n) ; \\
& \mathbf{D}(n+1)=\mathbf{A R}(n+1) \mathbf{B}+\mathbf{C R}(n+1) \mathbf{D})+\beta_{n} \mathbf{F}(n) ;
\end{aligned}
$$

\section{EndDo.}

Algorithm 2.4 (Matrix CRS2 method for solving (2.1))

1. Compute $\mathbf{R}(0)=\mathbf{E}-\mathbf{A X}(0) \mathbf{B}-\mathbf{C X}(0) \mathbf{D}$ for an initial guess $\mathbf{X}(0) \in \mathbf{R}^{\lambda m \times \lambda m}$. Set $\mathbf{R}^{*}(0)=\mathbf{P}(0)=\mathbf{U}(0)=\mathbf{R}(0)$.

2. Set $\mathbf{T}=\mathbf{A}^{T} \mathbf{R}^{*}(0) \mathbf{B}^{T}+\mathbf{C}^{T} \mathbf{R}^{*}(0) \mathbf{D}^{T}$.

3. For $n=0,1, \ldots$, until convergence Do:

$$
\mathbf{V}(n)=\mathbf{A} \mathbf{P}(n) \mathbf{B}+\mathbf{C P}(n) \mathbf{D}
$$




$$
\begin{aligned}
& \alpha_{n}=\langle\mathbf{R}(n), \mathbf{T}\rangle /\langle\mathbf{V}(n), \mathbf{T}\rangle ; \\
& \mathbf{Q}(n)=\mathbf{U}(n)-\alpha_{n} \mathbf{V}(n) ; \\
& \mathbf{X}(n+1)=\mathbf{X}(n)+\alpha_{n}(\mathbf{U}(n)+\mathbf{Q}(n)) ; \\
& \mathbf{W}(n)=\mathbf{A}(\mathbf{U}(n)+\mathbf{Q}(n)) \mathbf{B}+\mathbf{C}(\mathbf{U}(n)+\mathbf{Q}(n)) \mathbf{D} ; \\
& \mathbf{R}(n+1)=\mathbf{R}(n)-\alpha_{n} \mathbf{W}(n) ; \\
& \beta_{n}=\langle\mathbf{R}(n+1), \mathbf{T}\rangle /\langle\mathbf{R}(n), \mathbf{T}\rangle ; \\
& \mathbf{U}(n+1)=\mathbf{R}(n+1)+\beta_{n} \mathbf{Q}(n) ; \\
& \mathbf{P}(n+1)=\mathbf{U}(n+1)+\beta_{n}\left(\mathbf{Q}(n)+\beta_{n} \mathbf{P}(n)\right) ;
\end{aligned}
$$

\section{EndDo.}

From Algorithms 2.3 and 2.4 by using the equivalent relationships of periodic Sylvester matrix equation (1.1) and generalized Sylvester matrix equation (2.1) we can derive the CRS methods for solving periodic Sylvester matrix equation (1.1) as Algorithms 2.5 and 2.6 , respectively.

Algorithm 2.5 (Matrix CRS1 method for solving (1.1))

1. Choose $X_{j}(0) \in \mathbf{R}^{m \times m}$ for $j=1,2, \ldots, \lambda$ and set $X_{\lambda+1}(0)=X_{1}(0)$.

2. Compute $R_{j}(0)=E_{j}-A_{j} X_{j}(0) B_{j}-C_{j} X_{j+1}(0) D_{j}$ and set $R_{j}^{*}(0)=E_{j}(0)=R_{j}(0)$ for $j=1,2, \ldots, \lambda$. Let $E_{\lambda+1}(0)=E_{1}(0)$ and $R_{\lambda+1}^{*}(0)=R_{1}^{*}(0)$. Set $D_{j}(0)=A_{j} E_{j}(0) B_{j}+C_{j} E_{j+1}(0) D_{j}$. Let $\beta_{-1}=0$.

3. Set $T_{j}=A_{j}^{T} R_{j}^{*}(0) B_{j}^{T}+C_{j}^{T} R_{j+1}^{*}(0) D_{j}^{T}$ for $j=1,2, \ldots, \lambda$.

4. For $n=0,1, \ldots$, until convergence Do:

$$
S_{j}(n)=D_{j}(n)+\beta_{n-1}\left(F_{j}(n-1)+\beta_{n-1} S_{j}(n-1)\right) \text { for } j=1,2, \ldots, \lambda \text {. }
$$

Let $S_{\lambda+1}(n)=S_{1}(n)$;

$$
\begin{aligned}
& \alpha_{n}=\left(\sum_{j=1}^{\lambda}\left\langle R_{j}(n), T_{j}\right\rangle\right) /\left(\sum_{j=1}^{\lambda}\left\langle S_{j}(n), T_{j}\right\rangle\right) ; \\
& H_{j}(n)=E_{j}(n)-\alpha_{n} S_{j}(n) \quad \text { for } j=1,2, \ldots, \lambda ; \\
& F_{j}(n)=D_{j}(n)-\alpha_{n}\left(A_{j} S_{j}(n) B_{j}+C_{j} S_{j+1}(n) D_{j} \quad \text { for } j=1,2, \ldots, \lambda ;\right. \\
& X_{j}(n+1)=X_{j}(n)+\alpha_{n}\left(E_{j}(n)+H_{j}(n)\right) \text { for } j=1,2, \ldots, \lambda ; \\
& R_{j}(n+1)=R_{j}(n)-\alpha_{n}\left(D_{j}(n)+F_{j}(n)\right) \text { for } j=1,2, \ldots, \lambda ; \\
& \text { Let } R_{\lambda+1}(n+1)=R_{1}(n+1) ; \\
& \beta_{n}=\left(\sum_{j=1}^{\lambda}\left\langle R_{j}(n+1), T_{j}\right\rangle\right) /\left(\sum_{j=1}^{\lambda}\left\langle R_{j}(n), T_{j}\right\rangle\right) ; \\
& E_{j}(n+1)=R_{j}(n+1)+\beta_{n} H_{j}(n) \quad \text { for } j=1,2, \ldots, \lambda ; \\
& \left.D_{j}(n+1)=A_{j} R_{j}(n+1) B_{j}+C_{j} R_{j+1}(n+1) D_{j}\right)+\beta_{n} F_{j}(n) \text { for } j=1,2, \ldots, \lambda ;
\end{aligned}
$$

\section{EndDo.}


Algorithm 2.6 (Matrix CRS2 method for solving (1.1))

1. Choose $X_{j}(0) \in \mathbf{R}^{m \times m}$ for $j=1,2, \ldots, \lambda$ and set $X_{\lambda+1}(0)=X_{1}(0)$.

2. Compute $R_{j}(0)=E_{j}-A_{j} X_{j}(0) B_{j}-C_{j} X_{j+1}(0) D_{j}$ and set $R_{j}^{*}(0)=P_{j}(0)=U_{j}(0)=R_{j}(0)$ for $j=1,2, \ldots, \lambda$. Let $R_{\lambda+1}^{*}(0)=R_{1}^{*}(0)$.

3. Set $T_{j}=A_{j}^{T} R_{j}^{*}(0) B_{j}^{T}+C_{j}^{T} R_{j+1}^{*}(0) D_{j}^{T}$ for $j=1,2, \ldots, \lambda$.

4. For $n=0,1, \ldots$, until convergence Do:

$$
\begin{aligned}
& \text { Let } P_{\lambda+1}(n)=P_{1}(n) ; \\
& V_{j}(n)=A_{j} P_{j}(n) B_{j}+C_{j} P_{j+1}(n) D_{j} \quad \text { for } j=1,2, \ldots, \lambda ; \\
& \alpha_{n}=\left(\sum_{j=1}^{\lambda}\left\langle R_{j}(n), T_{j}\right\rangle\right) /\left(\sum_{j=1}^{\lambda}\left\langle V_{j}(n), T_{j}\right\rangle\right) ; \\
& Q_{j}(n)=U_{j}(n)-\alpha_{n} V_{j}(n) \quad \text { for } j=1,2, \ldots, \lambda ; \\
& X_{j}(n+1)=X_{j}(n)+\alpha_{n}\left(U_{j}(n)+Q_{j}(n)\right) \quad \text { for } j=1,2, \ldots, \lambda ; \\
& \text { Let } U_{\lambda+1}(n)=U_{1}(n) \quad \text { and } \quad Q_{\lambda+1}(n)=Q_{1}(n) ; \\
& W_{j}(n)=A_{j}\left(U_{j}(n)+Q_{j}(n)\right) B_{j}+C_{j}\left(U_{j+1}(n)+Q_{j+1}(n)\right) D_{j} \quad \text { for } j=1,2, \ldots, \lambda ; \\
& R_{j}(n+1)=R_{j}(n)-\alpha_{n} W_{j}(n) \quad \text { for } j=1,2, \ldots, \lambda ; \\
& \beta_{n}=\left(\sum_{j=1}^{\lambda}\left\langle R_{j}(n+1), T_{j}\right\rangle\right) /\left(\sum_{j=1}^{\lambda}\left\langle R_{j}(n), T_{j}\right\rangle\right) ; \\
& U_{j}(n+1)=R_{j}(n+1)+\beta_{n} Q_{j}(n) \quad \text { for } j=1,2, \ldots, \lambda ; \\
& P_{j}(n+1)=U_{j}(n+1)+\beta_{n}\left(Q_{j}(n)+\beta_{n} P_{j}(n)\right) \quad \text { for } j=1,2, \ldots, \lambda ;
\end{aligned}
$$

\section{EndDo.}

Based on the earlier discussion, we know that Algorithms 2.5 and 2.6 are just the matrix forms of the original CRS method. Hence, generally speaking, Algorithms 2.5 and 2.6 have the same properties as Algorithms 2.1 and 2.2. For instance, in exact arithmetic, Algorithms 2.5 and 2.6 will also terminate after a finite number of iterations.

\section{Numerical experiments}

In this section, we present two numerical examples to show the accuracy and efficiency of the proposed methods. We compare the performances of CRS methods to those of the CGS, BiCGSTAB [13], and CORS [16] methods.

In our experiments, all runs are started from the zero initial guess and implemented in MATLAB(R2014b) with a machine precision $10^{-16}$ on a personal computer with Intel(R) Core(TM) i7-6500U CPU $2.50 \mathrm{GHz} 2.60 \mathrm{GHz}, 16.0 \mathrm{~GB}$ memory.

Example 3.1 ([13]) Consider the periodic Sylvester matrix equation

$$
X_{j}+C_{j} X_{j+1} D_{j}=E_{j} \quad \text { for } j=1,2
$$




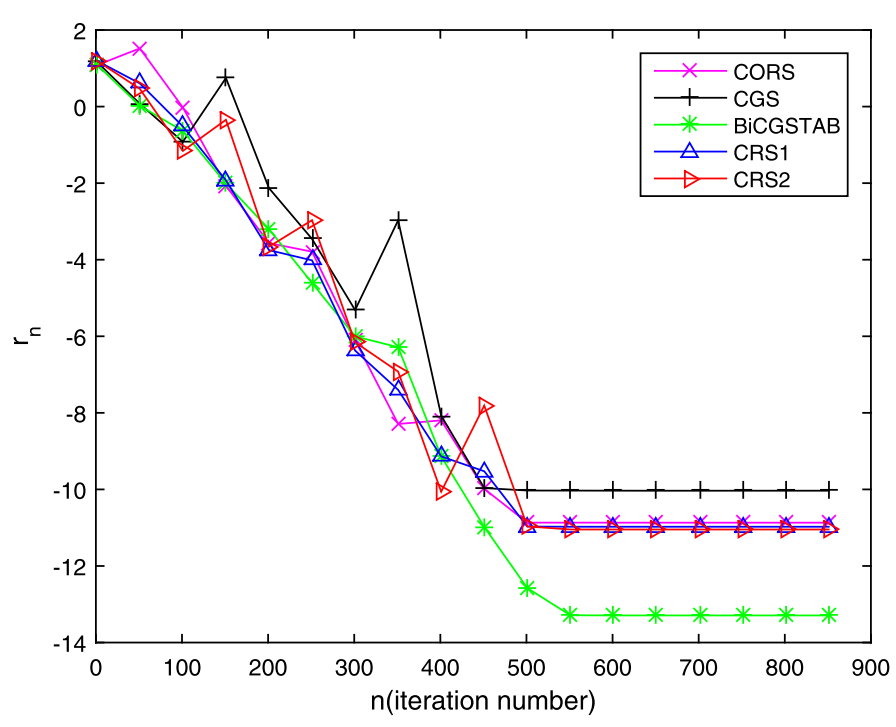

Figure 1 The residual for Example 3.1

with parameters

$$
\begin{aligned}
& C_{1}=\operatorname{tril}(\operatorname{rand}(m, m), 1)+\operatorname{diag}(2+\operatorname{diag}(\operatorname{rand}(m))), \\
& D_{1}=\operatorname{triu}(\operatorname{rand}(m, m), 1)+\operatorname{diag}(1.75+\operatorname{diag}(\operatorname{rand}(m))), \\
& C_{2}=\operatorname{triu}(\operatorname{rand}(m, m), 1)+\operatorname{diag}(1.75+\operatorname{diag}(\operatorname{rand}(m))), \\
& D_{2}=\operatorname{tril}(\operatorname{rand}(m, m), 1)+\operatorname{diag}(2+\operatorname{diag}(\operatorname{rand}(m))), \\
& E_{1}=E_{2}=\operatorname{rand}(m, m) .
\end{aligned}
$$

In this example, we set $m=20$. The numerical results are shown in Fig. 1, where

$$
r_{n}=\log _{10} \sqrt{\left\|E_{1}-X_{1}(n)-C_{1} X_{2}(n) D_{1}\right\|^{2}+\left\|E_{2}-X_{2}(n)-C_{2} X_{1}(n) D_{2}\right\|^{2}} .
$$

From Fig. 1 we find that the CRS1 and CRS2 methods are superior to the CGS and CORS methods, and the BiCGSTAB method is the best among them for Example 3.1. In addition, the residual history of the CRS1 method seems smoother than that of the CRS2 method.

Example 3.2 ([16]) Consider the periodic Sylvester matrix equations

$$
X_{j}+C_{j} X_{j+1} D_{j}=E_{j}, \quad \text { for } j=1,2
$$

with parameters

$$
\begin{aligned}
& C_{1}=-\operatorname{triu}(\operatorname{rand}(m, m), 1)+\operatorname{diag}(1.75+\operatorname{diag}(\operatorname{rand}(m))), \\
& D_{1}=\operatorname{tril}(\operatorname{rand}(m, m), 1)+\operatorname{diag}(1.25+\operatorname{diag}(\operatorname{rand}(m))), \\
& C_{2}=\operatorname{triu}(\operatorname{rand}(m, m), 1)+\operatorname{diag}(1.75+\operatorname{diag}(\operatorname{rand}(m))),
\end{aligned}
$$




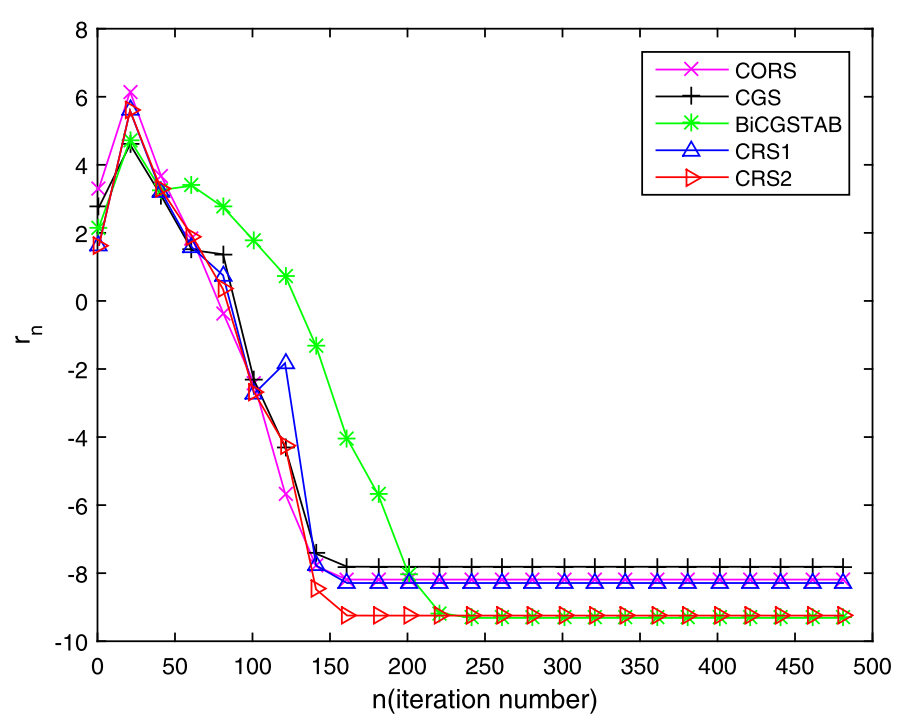

Figure 2 The residual for Example 3.2

$$
\begin{aligned}
& D_{2}=\operatorname{tril}(\operatorname{rand}(m, m), 1)+\operatorname{diag}(2+\operatorname{diag}(\operatorname{rand}(m))), \\
& E_{1}=E_{2}=\operatorname{rand}(m, m) .
\end{aligned}
$$

In this example, let $m=40$. The numerical results are shown in Fig. 2, where

$$
r_{n}=\log _{10} \sqrt{\left\|E_{1}-X_{1}(n)-C_{1} X_{2}(n) D_{1}\right\|^{2}+\left\|E_{2}-X_{2}(n)-C_{2} X_{1}(n) D_{2}\right\|^{2}} .
$$

From Fig. 2, for Example 3.2, we see that the CRS2 method is the best one among the five methods mentioned. The BiCGSTAB method can achieve higher accuracy than the CGS, CORS, and CRS1 methods.

\section{Conclusions}

In this paper, we present two matrix forms of the CRS iterative method for solving the periodic Sylvester matrix equation (1.1). Numerical examples and comparison with the CGS, BiCGSTAB, and CORS methods have illustrated that the CRS methods can work quite well in some situations. In addition, numerical results show that the CRS1 and CRS2 methods show different numerical performance, though they are mathematically equivalent.

Acknowledgements

The authors deeply thank the anonymous referees for helping to improve the original manuscript by valuable suggestions.

Funding

This work is supported by National Key Research and Development Program of China (No. 2018YFC0603500) and

National Science Foundation of China (Nos. 41725017, 41590864).

Competing interests

The authors declare that they have no competing interests. 


\section{Publisher's Note}

Springer Nature remains neutral with regard to jurisdictional claims in published maps and institutional affiliations.

\section{Received: 9 April 2018 Accepted: 21 February 2019 Published online: 04 March 2019}

\section{References}

1. Varga, A.: Periodic Lyapunov equations: some applications and new algorithms. Int. J. Control 67, 69-88 (1997)

2. Chu, E.K.W., Fan, H.-Y., Lin, W.-W.: Projected generalized discrete-time periodic Lyapunov equations and balanced realization of periodic descriptor systems. SIAM J. Matrix Anal. Appl. 29, 982-1006 (2017)

3. Liu, C., Peng, Y.J.: Stability of periodic steady-state solutions to a non-isentropic Euler-Maxwell system. Z. Angew. Math. Phys. 68, 105 (2017)

4. Zheng, X.X., Shang, Y.D., Peng, X.M.: Orbital stability of periodic traveling wave solutions to the generalized Zakharov equations. Acta Math. Sci. 37B, 1-21 (2017)

5. Zheng, X.X., Shang, Y.D., Di, H.F.: The time-periodic solutions to the modified Zakharov equations with a quantum correction. Mediterr. J. Math. 14, 152 (2017)

6. Tian, H.H., Han, M.A.: Bifurcation of periodic orbits by perturbing high-dimensional piecewise smooth integrable systems. J. Differ. Equ. 263(11), 7448-7474 (2017)

7. Liu, B.M., Liu, L.S., Wu, Y.H.: Existence of nontrivial periodic solutions for a nonlinear second order periodic boundary value problem. Nonlinear Anal. 72(7-8), 3337-3345 (2010)

8. Hao, X.N., Liu, L.S., Wu, Y.H.: Existence and multiplicity results for nonlinear periodic boundary value problems. Nonlinear Anal. 72(9-10), 3635-3642 (2010)

9. Liu, A.J., Chen, G.L.: On the Hermitian positive definite solutions of nonlinear matrix equation $X^{s}+\sum_{i=1}^{m} A_{i}^{*} X^{-t_{i}} A_{i}=Q$. Appl. Math. Comput. 243, 950-959 (2014)

10. Liu, A.J., Chen, G.L., Zhang, X.Y.: A new method for the bisymmetric minimum norm solution of the consistent matrix equations $A_{1} X B_{1}=C_{1}, A_{2} X B_{2}=C_{2}$. J. Appl. Math. 2013, Article ID 125687 (2013)

11. Stykel, T: Low-rank iterative methods for projected generalized Lyapunov equations. Electron. Trans. Numer. Anal. 30 187-202 (2008)

12. Bittanti, S., Colaneri, P.: Periodic Systems: Filtering and Control. Springer, London (2009)

13. Hajarian, M.: Matrix iterative methods for solving the Sylvester-transpose and periodic Sylvester matrix equations. J. Franklin Inst. 350, 3328-3341 (2013)

14. Hajarian, M.: Matrix form of biconjugate residual algorithm to solve the discrete-time periodic Sylvester matrix equations. Asian J. Control 20, 49-56 (2018)

15. LV, L.-L., Zhang, Z., Zhang, L., Wang, W.-S.: An iterative algorithm for periodic Sylvester matrix equations. J. Ind. Manag. Optim. 14, 413-425 (2018)

16. Hajarian, M.: Developing BiCOR and CORS methods for coupled Sylvester-transpose and periodic Sylvester matrix equations. Appl. Math. Model. 39, 6073-6084 (2015)

17. Kressner, D.: Large periodic Lyapunov equations: algorithms and applications. In: Proc. ECC03, Cambridge, UK, pp. 951-956 (2003)

18. Hajarian, M.: Solving the general Sylvester discrete-time periodic matrix equations via the gradient based iterative method. Appl. Math. Lett. 52, 87-95 (2016)

19. Hajarian, M.: Gradient based iterative algorithm to solve general coupled discrete-time periodic matrix equations over generalized reflexive matrices. Math. Model. Anal. 21, 533-549 (2016)

20. Hajarian, M.: A finite iterative method for solving the general coupled discrete-time periodic matrix equations. Circuits Syst. Signal Process. 34, 105-125 (2015)

21. Hajarian, M.: Developing CGNE algorithm for the periodic discrete-time generalized coupled Sylvester matrix equations. Comput. Appl. Math. 34, 755-771 (2015)

22. Hajarian, M.: Convergence analysis of the MCGNR algorithm for the least squares solution group of discrete-time periodic coupled matrix equations. Trans. Inst. Meas. Control 39, 29-42 (2017)

23. Cai, G.-B., Hu, C.-H.: Solving periodic Lyapunov matrix equations via finite steps iteration. IET Control Theory Appl. 6, 2111-2119 (2012)

24. Lv, L., Zhang, Z., Zhang, L.: A periodic observers synthesis approach for LDP systems based on iteration. IEEE Access 6 , 8539-8546 (2018)

25. Lv, L., Zhang, Z:: Finite iterative solutions to periodic Sylvester matrix equations. J. Franklin Inst. 354(5), 2358-2370 (2017)

26. Lv, L., Zhang, Z., Zhang, L.: A parametric poles assignment algorithm for second-order linear periodic systems. J. Franklin Inst. 354, 8057-8071 (2017)

27. Lv, L., Zhang, L.: Robust stabilization based on periodic observers for LDP systems. J. Comput. Anal. Appl. 20, 487-498 (2016)

28. Lv, L., Zhang, L.: On the periodic Sylvester equations and their applications in periodic Luenberger observers design. J. Franklin Inst. 353, 1005-1018 (2016)

29. Sogabe, T., Fujino, S., Zhang, S.-L.: A product-type Krylov subspace method based on conjugate residual method for nonsymmetric coefficient matrices. Trans. IPSJ 48, 11-21 (2007)

30. Zhang, L.-T., Zuo, X.-Y., Gu, T.-X., Huang, T.-Z., Yue, J.-H.: Conjugate residual squared method and its improvement for non-symmetric linear systems. Int. J. Comput. Math. 87, 1578-1590 (2010)

31. Chen, C.-R., Ma, C.-F.: A matrix CRS iterative method for solving a class of coupled Sylvester-transpose matrix equations. Comput. Math. Appl. 74, 1223-1231 (2017)

32. Zhang, L.-T., Huang, T.-Z., Gu, T.-X., Zuo, X.-Y.: An improved conjugate residual squared algorithm suitable for distributed parallel computing. Microelectron. Comput. 25, 12-14 (2008) (in Chinese)

33. Zhao, J., Zhang, J.-H.: A smoothed conjugate residual squared algorithm for solving nonsymmetric linear systems. In: 2009 Second International Conference on Information and Computing Science, ICIC, vol. 3, pp. 364-367 (2009)

34. Zuo, X.-Y., Zhang, L.-T., Gu, T.-X.: An improved generalized conjugate residual squared algorithm suitable for distributed parallel computing. J. Comput. Appl. Math. 271, 285-294 (2014) 
35. Sogabe, T., Zhang, S.-L.: Extended conjugate residual methods for solving nonsymmetric linear systems. Numerical Linear Algebra and Optimization 88-99 (2003)

36. Sogabe, T., Sugihara, M., Zhang, S.-L:: An extension of the conjugate residual method to nonsymmetric linear systems. J. Comput. Appl. Math. 226, 103-113 (2009)

37. Saad, Y.: Iterative Methods for Sparse Linear Systems, 2nd edn. SIAM, Philadelphia (2003)

Submit your manuscript to a SpringerOpen ${ }^{\circ}$ journal and benefit from:

- Convenient online submission

$\checkmark$ Rigorous peer review

- Open access: articles freely available online

- High visibility within the field

- Retaining the copyright to your article

Submit your next manuscript at $\boldsymbol{~ s p r i n g e r o p e n . c o m ~}$ 